\title{
Estado actual en la legislación autonómica de los árboles singulares:del concepto a la protección
}

\section{Current status in the autonomous legislation of singular trees: from concept to protection}

\author{
Villota Gálvez, M. ${ }^{1 *}$ \\ ${ }^{1}$ Grupo de Ecología y Paisaje. Departamento de Ingeniería y Gestión Forestal y Ambiental. \\ Escuela Técnica Superior de Ingenieros de Montes, Forestal y del Medio Natural, \\ Universidad Politécnica de Madrid, Avda. de las Moreras, Ciudad Universitaria, s/n, 28040 - Madrid \\ *Autor para correspondencia: martavillota@mvpaisaje.com
}

\section{Resumen}

Entender el árbol como elemento natural y cultural en sus múltiples realidades viene siendo un objeto de investigación cuyo alcance se complica desde el intento de establecer su definición. La figura jurídica "árbol singular" sirve para reconocer la importancia del arbolado de interés y constituye el instrumento para legitimarlo como patrimonio de la sociedad. Sin embargo, la heterogeneidad de criterios y tipologías que caracteriza la legislación de las diferentes Comunidades Autónomas dificulta su aplicación. El artículo se presenta como una discusión que indaga en el marco jurídico del patrimonio arbóreo a nivel autonómico y estatal, expone una revisión a partir de previas investigaciones con el fin de contrastar la normativa y conocer su estado actual

\section{Summary}

Understanding the tree as a natural and cultural element in its multiple realities has been an object of research whose scope is complicated by the attempt to establish its definition. The legal figure "singular tree" serves to recognize the importance of the trees of interest and constitutes the instrument to legitimize it as the patrimony of society. However, the heterogeneity of criteria and typologies that characterize the legislation of the different Autonomous Communities makes their application difficult. The article is presented as a discussion that investigates the legal framework of arboreal heritage at the regional and state levels, exposes a review from previous investigations in order to contrast the regulations and know their current status.

Palabras clave: legislación, árbol singular, conservación, patrimonio arbóreo

Keywords: legislation, singular tree, conservation, arboreal heritage 


\section{Introducción}

Los árboles y las arboledas que destacan por su valor, por su singularidad y por determinadas cualidades de interés excepcional, constituyen un conjunto de bienes de la comunidad donde se encuentran, reconociéndose como patrimonio de la sociedad. Tanto generadores de biodiversidad como productores del espacio habitado y humano se integran dentro del patrimonio natural y cultural desde una perspectiva ecológica, histórica y paisajística. Sin embargo, buena parte de este legado se encuentra amenazado por diversas causas, la mayoría de las veces, abandono u olvido, desconocimiento de su existencia y de su importancia. La desaparición de un elevado número de árboles situados en zonas rurales se debe sobre todo a la mecanización de la agricultura que trajo los cambios en el modelo económico. La ausencia de un estado legal más concreto del arbolado junto a las políticas agrarias comunitarias, no favorece su preservación (Villota, 2015). Hoy en día, la sociedad va tomando conciencia de este problema y la preocupación por la conservación de la naturaleza y, en particular, del patrimonio arbóreo ha impulsado numerosos estudios, catálogos e inventarios, normas y nuevas figuras de protección del arbolado en la legislación ambiental, e incluso leyes propias en distintas Comunidades Autónomas (en adelante CC.AA.)

La conservación de estos árboles singulares se posibilita desde su designación bajo las figuras de protección recogidas en la legislación correspondiente en cada caso. No obstante, la norma no profundiza en las cualidades "únicas" y "extraordinarias" que ha de reunir cada ejemplar o arboleda para ser designado como singular. Los aspectos a los que se alude en las diferentes definiciones que se hace del término o figura de protección, no suelen describirse y, en cualquier caso, quedan reflejados en una escueta mención. La ambigüedad que existe en torno a la definición induce a diferentes interpretaciones y nomenclaturas (Árbol Singular, Monumental, Excepcional, Notable, Monumento Natural, etc.) en un intento de delimitar la categoría para regularlo jurídicamente (Villota, 2015). Abordar la revisión de la legislación, cara a una futura actualización, así como la promoción de otras figuras de arbolado de interés desde las ordenanzas y la normativa local, proporcionaría concreción y mayor agilidad.

Numerosos artículos se centran en casos de estudio sobre arbolado en zonas concretas, o bien tratan sobre los catálogos regionales, o sobre legislación en materia de protección de arbolado de un modo meramente descriptivo. La exposición de antecedentes históricos transita sobre la evolución normativa, con frecuencia, sin llegar al análisis crítico de la situación actual. Tras la revisión de publicaciones sobre estas cuestiones, no parece hallarse, pues, bibliografía actualizada al respecto. sobre todo, en lo que se refiere al estudio comparativo entre normas dentro del panorama autonómico actual.

Así pues, el presente artículo se estructura en un análisis de la legislación autonómica, tratando la cuestión de la definición, a partir de la bibliografía relevante sobre el tema, con el objeto de profundizar en el actual marco normativo del arbolado singular a través de una comparativa entre las diversas nociones (figuras y 
catálogos) y condiciones de regulación propuestas por las distintas leyes. El estudio se desarrolla en base a los siguientes objetivos:

I. Dar cuenta de la situación de la normativa referente a la figura "Árbol singular" a nivel estatal.

II. Conocer los criterios que sustentan la definición de árbol singular, identificando las particularidades de cada comunidad autónoma, dando cuenta así de la imprecisión sobre el concepto global.

\section{HACIA UNA DEFINICIÓN, HACIA UNA CONSERVACIÓN}

Gran parte de las definiciones para el arbolado se centran en la visión más descriptiva y funcional del mismo como fuente de recursos y energía, obviando otros aspectos referidos al significado simbólico o cultural, así como en el aspecto paisajístico. Es precisa una definición clara del recurso inventariado para garantizar la congruencia y la comparación con otros estudios y facilitar la comunicación de los resultados (Kleinn, 2000). Apoyándose en otras investigaciones y en su propia experiencia, el botánico Francis Hallé aporta una extensa explicación sobre el árbol en su ensayo Plaidoyer pour l'arbre (Hallé, 2005), definición que más tarde cuestiona (Hallé, 2011) ante la dificultad por la multiplicidad de enfoques. Como aque1la descripción, existen numerosas denominaciones desde el punto de vista anatómico y funcional del árbol. Así mismo, Ceballos y Ruiz de la Torre definen "árbol" diferenciándolo de "arbolillo" bajo una descripción basada en las propiedades estructurales del mismo (Ceballos y Ruiz de la Torre, 1979). Ahora bien, más allá de ser el recurso leñoso o productivo perteneciente a los sistemas y poblaciones forestales, la complejidad se incrementa al tratar de estimar la importancia del árbol y caracterizarlo con respecto a una situación local, tarea cuyo objeto viene a ser la identificación de elementos como "árbol singular" con el fin de su conservación (Villota, 2015).

Alejándose del concepto más utilitario y funcional del árbol que lo describe desde su fisiología, se enuncian otras definiciones referidas a su significación cultural. Significado que adquieren aquellos árboles, en relación con la historia o la mitología que les envuelve, a los que se vinculan los pueblos desde antaño. De este modo, diversos árboles han sido centro de la actividad de comunidades, estableciéndose vínculos entre sí a través de las fiestas, los ritos y las tradiciones que se celebraban al amparo de la sombra de su copa. El simbolismo religioso o espiritual, el carácter popular o el significado político de árboles junteros o juraderos como jueces y testigos de la historia, les confiere un sentido cultural muy profundo, representantes de una identidad local o regional, en ocasiones, alcanzando el reconocimiento territorial, como ocurre con el Roble de Guernica (árbol sagrado, representante de lo jurídico y lo político de toda una ciudadanía). En torno al concepto de árbol sagrado, precisamente es en el País Vasco donde la tradición del olentzero en 
Navidad se presenta como otra expresión de culto al árbol asociada a estas fiestas (Caro Baroja, 1946). Así mismo, fiestas y otras celebraciones como es la Fiesta del Árbol o el Mayo, evidencian el significado folclórico o costumbrista que adquiere el árbol, cuya definición se construye, por tanto, desde un enfoque absolutamente cultural. Una de las definiciones que abarca un significado integral es la que sirve para describir a los "árboles viejos", también llamados "antiguos" o "venerables", por su enorme valor ecológico y simbólico o cultural. Estos son, con frecuencia, aquellos árboles trasmochados (o sometidos a podas drásticas), objeto, en general, de una antigua gestión del monte que ha sido abandonada, derivando en un paisaje que hoy es legado cultural.

Desde las definiciones que nos aportan los diccionarios, la definición de "singularidad" que ofrece el Diccionario de la Naturaleza, es "condición de fuera de lo común. La singularidad de los rasgos bióticos o abióticos de un territorio es una de las razones importantes para su conservación." El mismo diccionario describe "monumento natural" como la característica natural única, de gran interés para la ciencia, la cultura o la educación (Ramos (coord.), 1987). Aspectos similares - "valores científicos, culturales o paisajísticos"- son destacados por la Ley 4/1989 de Conservación de los Espacios Naturales de la Flora y Fauna Silvestres, en la definición de Monumento Natural (igualmente por la ley que la deroga, Ley 42/2007 del Patrimonio Natural y la Biodiversidad). Con dicho término, "monumento natural", se sirven las leyes ambientales para referirse a aquellos elementos más raros o singulares de la naturaleza. Se trata entonces de una noción más amplia y genérica que aún no profundiza en la idiosincrasia del arbolado. Es necesario llegar a la normativa autonómica para encontrarse con vocablos más específicos.

\section{Metodología}

El objeto de estudio en esta publicación parte de previos procesos de investigación que se inician en la observación de diversos árboles de interés ubicados en los paisajes de Álava (Villota, 2015). El trabajo revisa la situación de la normativa estatal a través de la normativa autonómica competente. En un recorrido que abarca la totalidad de las CC.AA. se exploran las distintas medidas de carácter reglamentario, las cuales se establecen según competencias (política ambiental, forestal, territorial, urbanística, etc.) y según los aspectos a regular del arbolado. El ámbito temporal del estudio comprende desde 1986, momento en que se aprueba el primer catálogo de árboles singulares con valor legal en España, hasta la actualidad.

En la metodología empleada se optó por un análisis cualitativo de contenido, en cuanto a los componentes que se ponen en relación dentro de cada disposición legal (leyes, ordenanzas, órdenes o decretos) correspondiente a cada comunidad autónoma. Se recopilaron y seleccionaron textos y artículos que tratan sobre la legislación del patrimonio natural, especialmente sobre arbolado y su evolución histórica. Sobre una documentación bibliográfica, recogida desde las fuentes oficiales 
Tabla 1. Cuadro resumen de la situación actual en la legislación autonómica.

\begin{tabular}{|c|c|c|c|}
\hline CC.AA & $\begin{array}{l}\text { ¿Disfruta de una } \\
\text { ley de patrimonio } \\
\text { arbóreo? }\end{array}$ & $\begin{array}{l}\text { ¿Qué figura/s de protección } \\
\text { posee para árbol singular? } \\
\text { Otras figuras anteriores }\end{array}$ & $\begin{array}{c}\text { ¿Crea un Catálogo } \\
\text { o Inventario } \\
\text { de Árboles Singulares? } \\
\text { [Denominación] si al menos } \\
\text { se dicta su creación }\end{array}$ \\
\hline Andalucía & NO & $\begin{array}{l}\text { Nonumento Natural/ } \\
\text { Elementos vegetales } \\
\text { singulares del paisaje }\end{array}$ & $\begin{array}{c}\text { NO } \\
\text { [Catálogo Andaluz de Árboles } \\
\text { y Arboledas Singulares] }\end{array}$ \\
\hline Aragón & NO & Árbol y Arboleda Singular & $\begin{array}{l}\text { Catálogo de Árboles } \\
\text { Arboledas Singulares }\end{array}$ \\
\hline Asturias & NO & Monumento Natural & $\mathrm{NO}$ \\
\hline Cantabria & NO & $\begin{array}{c}\text { Árbol Singular/ } \\
\text { Monumento Natural/ }\end{array}$ & $\begin{array}{l}\text { Inventario de Árboles } \\
\text { Singulares de Cantabria }\end{array}$ \\
\hline $\begin{array}{l}\text { Castilla- } \\
\text { La Mancha }\end{array}$ & NO & $\begin{array}{c}\text { Árbol Singular/ } \\
\text { Monumento Natural/ }\end{array}$ & $\begin{array}{l}\text { NO [Inventario de Árboles } \\
\text { y Ejemplares Singulares] }\end{array}$ \\
\hline Castilla y León & NO & $\begin{array}{l}\text { Árboles Notables/ } \\
\text { Monumento Natural }\end{array}$ & \begin{tabular}{|c|} 
[Catálogo Regional de Árboles \\
Notables]/ Catálogo \\
Especímenes Vegetales \\
de singular relevancia \\
de Castilla y León
\end{tabular} \\
\hline Cataluña & NO & $\begin{array}{c}\text { Árbol o Arboleda } \\
\text { Monumental/ de interés } \\
\text { comarcal/ de interés local/ }\end{array}$ & $\begin{array}{c}\text { Inventario } \mathrm{AM}, \mathrm{DM} \text {. } \\
\text { Inventario } \mathrm{AC}, \mathrm{DC} \\
\text { y } \mathrm{AL}, \mathrm{DL}\end{array}$ \\
\hline Extremadura & NO & $\begin{array}{c}\text { Árbol Singular/ } \\
\text { Monumento Natural/ }\end{array}$ & $\begin{array}{c}\text { Declaración } \\
\text { de Árboles Singulares }\end{array}$ \\
\hline Galicia & NO & $\begin{array}{l}\text { Árbol y Arboleda Singular/ } \\
\text { Monumento Natural/ }\end{array}$ & $\begin{array}{l}\text { Catálogo Gallego } \\
\text { de Árboles Singulares }\end{array}$ \\
\hline Islas Baleares & SI & Árbol Singular & $\begin{array}{l}\text { Catálogo de Árboles } \\
\text { Singulares }\end{array}$ \\
\hline Islas Canarias & NO & Monumento Natural & NO \\
\hline La Rioja & NO & Árboles Singulares & $\begin{array}{c}\text { Catálogo } \\
\text { de Árboles Singulares }\end{array}$ \\
\hline Madrid & SI & Árbol Singular & $\begin{array}{c}\text { Catálogo } \\
\text { Árboles Singulares }\end{array}$ \\
\hline Murcia & SI & $\begin{array}{c}\text { Árbol Monumental/ Árbol } \\
\text { Singular/ Árbol Monumental } \\
\text { de Interés Local }\end{array}$ & $\begin{array}{l}\text { NO [Catálogo de Árboles } \\
\text { Monumentales y Singulares } \\
\text { bajo protección genérica] }\end{array}$ \\
\hline Navarra & NO & Monumento Natural & $\begin{array}{c}\text { Declaración de } \\
\text { Monumentos naturales }\end{array}$ \\
\hline País Vasco & NO & Árbol Singular & $\begin{array}{c}\text { Catálogo de } \\
\text { Árboles Singulares }\end{array}$ \\
\hline Valencia & SI & $\begin{array}{c}\text { Árbol Monumental/ Árbol } \\
\text { Singular/ Árbol Monumental } \\
\text { de Interés Local }\end{array}$ & $\begin{array}{c}\text { NO [Catálogo de Árboles } \\
\text { Monumentales y Singulares bajo } \\
\text { protección genérica] }\end{array}$ \\
\hline
\end{tabular}


Tabla 2. Aspectos contemplados en la definición de Árbol Singular.

\begin{tabular}{|c|c|c|c|c|c|c|c|c|c|}
\hline \multirow[b]{2}{*}{ CC.AA. } & \multicolumn{9}{|c|}{ Aspectos/ criterios que contempla la definición } \\
\hline & 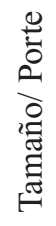 & $\overrightarrow{\tilde{J}}$ & $\begin{array}{l}\widetilde{N} \\
\stackrel{\Xi}{\sharp} \\
\simeq\end{array}$ & 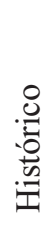 & 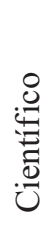 & 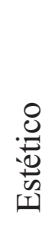 & 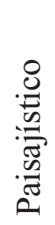 & 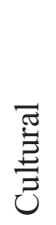 & : \\
\hline Andalucía & $\mathrm{X}$ & $\mathrm{X}$ & $\mathrm{X}$ & $\mathrm{X}$ & & $\mathrm{X}$ & $\mathrm{X}$ & $\mathrm{X}$ & \\
\hline Aragón & $\mathrm{X}$ & $\mathrm{X}$ & $\mathrm{X}$ & $\mathrm{X}$ & $\mathrm{X}$ & & & $\mathrm{X}$ & \\
\hline Asturias & & & $\mathrm{X}$ & & & $\mathrm{X}$ & & & \\
\hline Cantabria & $\mathrm{X}$ & $\mathrm{X}$ & $\mathrm{X}$ & & & $\mathrm{X}$ & & & \\
\hline Castilla-La Mancha & $\mathrm{X}$ & $\mathrm{X}$ & $\mathrm{X}$ & $\mathrm{X}$ & $\mathrm{X}$ & $\mathrm{X}$ & & $\mathrm{X}$ & $\mathrm{X}$ \\
\hline Castilla y León & & & & & & & & & $\mathrm{X}$ \\
\hline Cataluña & $\mathrm{X}$ & $\mathrm{X}$ & & $\mathrm{X}$ & $\mathrm{X}$ & $\mathrm{X}$ & $\mathrm{X}$ & $\mathrm{X}$ & $\mathrm{X}$ \\
\hline Extremadura & $\mathrm{X}$ & $\mathrm{X}$ & $\mathrm{X}$ & $\mathrm{X}$ & $\mathrm{X}$ & $\mathrm{X}$ & & $\mathrm{X}$ & \\
\hline Galicia & & & & & $\mathrm{X}$ & $\mathrm{X}$ & $\mathrm{X}$ & $\mathrm{X}$ & \\
\hline Islas Baleares & $\mathrm{X}$ & & & & $\mathrm{X}$ & & & $X$ & \\
\hline Islas Canarias & & & $\mathrm{X}$ & & & $\mathrm{X}$ & & & \\
\hline La Rioja & $\mathrm{X}$ & $\mathrm{X}$ & & & & $\mathrm{X}$ & $\mathrm{X}$ & & \\
\hline Madrid & $X$ & $\mathrm{X}$ & $\mathrm{X}$ & $\mathrm{X}$ & $\mathrm{X}$ & $\mathrm{X}$ & & $\mathrm{X}$ & $\mathrm{X}$ \\
\hline Murcia & $\mathrm{X}$ & $\mathrm{X}$ & $\mathrm{X}$ & $\mathrm{X}$ & $\mathrm{X}$ & & $\mathrm{X}$ & $\mathrm{X}$ & \\
\hline Navarra & $\mathrm{X}$ & $\mathrm{X}$ & $\mathrm{X}$ & $\mathrm{X}$ & $\mathrm{X}$ & $\mathrm{X}$ & $\mathrm{X}$ & $\mathrm{X}$ & \\
\hline País Vasco & $\mathrm{X}$ & $X$ & & $\mathrm{X}$ & & $\mathrm{X}$ & $\mathrm{X}$ & & \\
\hline Valencia & $\mathrm{X}$ & $\mathrm{X}$ & $\mathrm{X}$ & $\mathrm{X}$ & $\mathrm{X}$ & & $\mathrm{X}$ & $\mathrm{X}$ & \\
\hline
\end{tabular}

de cada una de las Administraciones y del Boletín Oficial del Estado, se constató la normativa oportuna y se cotejaron los componentes que se describen:

- La figura de protección: las definiciones referidas a la figura legal que determina la categoría de máxima protección que puede alcanzar un árbol según condiciones específicas (criterios).

- Y la creación del Catálogo o el Inventario: el instrumento público de carácter administrativo en el que se inscriben, describen y, en su caso, regulan y tutelan los elementos arbóreos, esto es, si constituyen la vía para configurar un régimen de protección específico.

En base al número de CC.AA. que, desde las políticas ambientales que emprenden, consideran la protección de árboles y/o arboledas monumentales, el análisis también es cuantitativo. Se hace un balance de aquellas comunidades que establecen la categoría de "árbol singular" e, incluso, contienen leyes específicas para la 
conservación, protección y regulación del patrimonio arbóreo, y aquellas que, sin figura o ley, al menos, albergan la posibilidad de regularlo a través de instrumentos u otras figuras de protección similar (Tabla 1). Además se comparan los criterios propuestos en sus definiciones (Tabla 2).

El presente artículo recoge una exposición deteniéndose en aquellos puntos de mayor interés, esto es, sobre aquellas autonomías cuya normativa analizada abren mayor discusión. A partir del marco teórico inicial, la metodología sirve para dirigir las preguntas acerca del proceso que se plantea desde la concepción de árbol singular hasta la figura legal que establece su reconocimiento lícito.

\section{Análisis, resultados y discusión}

Dentro del marco legislativo y en lo que respecta a la cuestión del arbolado, a su calificación y a sus modos de agrupación, nos encontramos expresiones tales como árboles «notables», «excepcionales», «sobresalientes», que suelen aparecer en distintas órdenes reguladoras de Catálogos sobre especies merecedoras de especial protección. Las CC.AA. se referirán, en su mayoría, a árboles monumentales o árboles singulares. Un árbol será singular bajo multitud de aspectos, algunos de los cuales podrán seleccionarse y desarrollarse para ser utilizados como potenciales criterios en la evaluación del arbolado (Villota y Escribano, 2015). La noción de árbol singular se funda en diferentes definiciones según diversas cualidades (edad, tamaño, rareza, belleza, paisaje, historia, etc.) que, sin embargo, no se especifican detalladamente en la legislación. Definir un concepto común para patrimonio arbóreo se complica desde la multiplicidad de las distintas Autonomías. La falta de consenso y el contraste entre sí dificulta la determinación de criterios para su evaluación. Además de las contradicciones existentes por superposición de figuras de protección según su reglamentación sectorial. La revisión de este marco jurídico pone de manifiesto ciertas incongruencias, como se verá a continuación.

\section{ANALISIS COMPARATIVO A TRAVÉS DEL CONCEPTO Y LA CATALOGACIÓN}

A nivel estatal, la figura de protección de árbol singular no se menciona de manera específica, solo se hace referencia a su protección desde una categoría genérica que puede ser la que se establece como "monumento natural". Así es como queda definida en la Ley 4/1989 de Conservación de los Espacios Naturales y de la Flora y Fauna Silvestres, ley que, en parte procede de la Ley de 1975 de Espacios Naturales Protegidos, y a las sucesivas modificaciones de aquélla. La Ley 42/2007, del Patrimonio Natural y de la Biodiversidad, que viene a derogar y sustituir a la Ley 4/1989, amplía aquella definición incluyendo al árbol singular. Entre los principios que inspiran aquella ley, subrayamos aquellos que se centran "en la preservación de la variedad, singularidad y belleza de los ecosistemas naturales, de la diversidad geológica y del paisaje". Estas cualidades apenas son reflejadas en lo 
que respecta a las políticas de protección ambiental, pero son pautas para nuevos parámetros o conceptos en la evaluación del patrimonio natural.

A continuación, se hace un repaso por la legislación vigente autonómica y más representativa contrastando las diferentes definiciones que se presentan en torno al concepto de árbol singular. A excepción de unas pocas CC.AA. (Madrid, Comunidad Valenciana, Islas Baleares, y recientemente, Murcia) que poseen su propia norma de patrimonio arbóreo, la mayoría de las administraciones basa la regulación de dicho patrimonio en otras normas autonómicas, e incluso estatales, de protección más general (leyes ambientales o de conservación de espacios naturales, leyes forestales, etc.) que sustentan entre sus artículos la figura legal destinada al arbolado singular.

Entre las CC.AA., son numerosas aquellas que expresan la protección a través de la figura de Monumento Natural, noción genérica y, en parte, obsoleta, pues apenas se ha modificado con respecto a la definición que se propone originalmente en la Ley 4/1989, resumiéndose en términos como formaciones de notoria singularidad, rareza o belleza, que merecen ser objeto de una protección especial. A pesar de la amplitud de la concepción, no hay acuerdo general ni criterios claros a la hora de su utilización. (Florido, G., Lozano, P.J., 2005). Son los casos de Asturias, Islas Canarias y Navarra que, sin explicar mucho más, sugieren así la protección arbórea. En las dos primeras no existe catálogo de árboles singulares. Navarra, al menos, destaca la importancia de los árboles o grupos de árboles — por sus características, edad, historia o dimensiones, particularidad científica, interés ecológico, paisajístico o cultural - y los declara como "monumentos naturales" a través de sucesivos decretos para la protección de los mismos. Constituye, así lo que podría acercarse a un catálogo.

Del mismo modo, la figura Monumento Natural es utilizada por otros territorios autonómicos como Cantabria, Castilla-La Mancha, Castilla y León y Andalucía (entre otras), generalmente recogida bajo la categoría de espacios naturales protegidos en leyes de conservación de la naturaleza (derivadas de aquella Ley 4/1989). A esta situación se le añade la creación de nuevos vocablos que emergen posteriormente, duplicándose las funciones de protección, como se verá a continuación.

A falta de una figura que contenga claramente la definición de "árbol singular" (o similar), en ocasiones, esta se enuncia a través de la formulación de un Inventario o Catálogo de Árboles Singulares. La descripción del mismo, en la orden o decreto correspondiente a la comunidad autónoma, expone las cualidades o circunstancias que deben cumplir los ejemplares para ser incluidos en el catálogo que se presenta. Así, Cantabria, como pionera en la conservación de los árboles singulares, aprobó el Inventario Abierto de Árboles Singulares (por Orden de 28 de mayo de 1986) conforme al Decreto 82/1985 por el que se aprueba el Reglamento de la Ley de Cantabria 6/1984 sobre Protección y Fomento de las Especies Forestales Autóctonas. Allí se incluían todos aquellos ejemplares que se consideren excepcionales por su belleza, porte, longevidad, especie o cualquier otra circunstancia que lo aconseje, definición que sigue sin profundizar más de lo que ya detallaba la ley estatal. Y que se apoya, por tanto, en la figura de "monumento natural" que mantie- 
ne en su correspondiente ley de conservación de la naturaleza (Ley 4/2006). Prácticamente igual a este enunciado, así responde la definición que recoge la legislación vigente de Castilla-La Mancha, Ley 3/2008, de 12 de junio, de Montes y Gestión Forestal Sostenible, añadiendo el matiz de rareza, interés cultural, histórico o científico. Es como dicha ley delimita los ejemplares que serán declarados singulares y se inscribirán en el Inventario de Árboles y Ejemplares Singulares de Castilla-La Mancha. Pero lo cierto es que, salvo contadas iniciativas de inventarios a nivel provincial, local o regional, en Castilla-La Mancha no se ha configurado un verdadero Catálogo de árboles singulares, tan solo dos ejemplares fueron declarados (uno ya desaparecido).

La definición podría ajustarse mejor en el caso de Castilla y León, que incorpora la figura "árbol notable" a través de la última ley aprobada, Ley 4/2015, del Patrimonio Natural (que modifica la anterior, Ley 8/1991, de Espacios Naturales) y dicta la creación del Catálogo Regional de Árboles Notables. Sin embargo, esta nueva voz, "árboles notables" (integrada dentro de la Red de Zonas Naturales de Interés Especial) se apoya en una vaga expresión pues son aquellos ejemplares, individuales o agrupados, que sean declarados como tales por entenderse que deben ser dotados de un régimen de protección especial, en atención a sus características singulares. Nos preguntamos qué se entiende por dichas características singulares y dónde se especifican. Una definición que incluye el término que define. Al igual que en anteriores casos, tampoco está claro de qué modo se evita la superposición de funciones: la que cumple esta figura (árbol notable) y la de Monumento Natural, dentro de la Red de Espacios Naturales Protegidos (REN) de la Ley 4/2015 y que suponemos referida a otros elementos.

Sucede como en Extremadura donde, regulando ambas figuras: Monumento Natural y Árbol Singular dentro de la misma ley (Ley 9/2006, en su artículo 16 sobre la tipología de los espacios naturales protegidos), se reserva la primera para la calificación exclusiva de elementos abióticos y se utiliza la de Árbol Singular para las especies vegetales, duplicando así la tipología de clasificación (Florido, G., Lozano, P.J., 2005). De cualquier forma, hay cierta coherencia en cuanto que se dictó el Decreto 4/1999, para la declaración de árboles singulares, en desarrollo a la Ley 8/1998 (modificada por la Ley 9/2006), al año siguiente. En el caso de Extremadura, lo interesante, además, es que, a través de este mismo Decreto, determina los criterios para desarrollar la figura de "árbol singular" categoría incluida dentro de los espacios naturales protegidos, en la Ley 8/1998. En el artículo 4 de dicho Decreto se establecen criterios de "singularidad" (referidos a la rareza, tanto de la especie o del ejemplar, como del emplazamiento, incluye el valor paisajístico y estético); "biométricos" (edad, diámetros y alturas) e "históricos y culturales" (en relación con eventos históricos o fenómenos culturales). Asimismo, define otros criterios para establecer el área periférica de protección, donde se insiste en la importancia paisajística del lugar. El Decreto aporta además una serie de características que han de cumplir los ejemplares para ser considerados "Árboles Singulares", definición que presenta en su primer artículo y que conviene distinguir de los criterios de evaluación para su "declaración" (en artículo 4). Sin llegar a formalizar 
un Catálogo, sí se han declarado decenas de árboles singulares en este y sucesivos decretos.

Similar a Extremadura es la situación de Galicia, en cuanto a que regula ambas figuras de protección (monumento natural y árbol singular) que proceden de una misma ley de conservación de la naturaleza. A partir de la Ley 9/2001, que alberga la figura de "Monumento natural", crea el Catálogo gallego de Árboles Singulares de Galicia, para asegurar la conservación de "aquellos ejemplares o rodales que, por sus valores o interés natural, cultural, científico, educativo, estético o paisajístico", se incluyen en el mismo. En virtud de esta ley 9/2001 se aprueba el Decreto 67/2007 para la aprobar y regular dicho Catálogo. Es entonces cuando concede un artículo a la definición de árbol singular bajo el título "Árboles y formaciones singulares" donde describe brevemente los árboles y otras formaciones de especies autóctonas o foráneas que, según características excepcionales, tienen consideración de ser singulares.

Sorprendente es la Comunidad de Andalucía, que regula y desarrolla la figura de Monumento natural por Decreto 225/1999, donde incluye a los “árboles centenarios, históricos o monumentales" en la categoría de "Monumentos Naturales de carácter Biótico" (según los tipos descritos en su artículo 4). Figura de protección que se mantiene hasta hoy sin modificación de aquel decreto. Por si esto fuera poco, desarrolla un nuevo término: "elementos vegetales singulares del paisaje" en la Ley 8/2003 para la protección de hábitats y otros elementos del paisaje donde incluye a los árboles y arboledas singulares, a cuyo efecto crea el Catálogo Andaluz. Casi diez años más tarde, se aprueba el Decreto 23/2012, en base a aquella ley, que desarrolla el Catálogo Andaluz de Árboles y Arboledas Singulares) La definición de árbol o arboleda singular viene a ser aquel "elemento singular del paisaje" declarado que cumple con las singularidades detalladas: morfológicas, dendrométricas, belleza, longevidad, rareza, históricos y culturales, y paisajístico. Solo a través de una Orden que establece el régimen de protección aplicable se efectúa la declaración (de árbol singular). Sin haberse aprobado aún dicha orden, el inventario actualmente conocido de árboles y arboledas singulares -realizado por la Junta de Andalucía y mediante participación ciudadana- no deja de ser, por tanto, una colección de árboles que dista del significado real, propio del Catálogo creado en la legislación.

El desconcierto y la repetición ante la profusión de figuras de protección se hace evidente en estas Comunidades Autónomas. Como se ha observado, establecen un término específico para la protección de sus árboles singulares o lo hacen a través de un inventario. Al margen de la aportación fructífera que esto conlleva como vías hacia una conservación del arbolado, el hecho es que estos instrumentos coexisten con las figuras pertenecientes a la red de espacios naturales protegidos ("monumento natural") propuestas desde sus leyes de conservación de la naturaleza, lo que genera cierto conflicto.

La cuestión podría aclararse remitiéndose a la ley de conservación que recoge la figura de "monumento natural", como prescribe la normativa de la Comunidad Aragón, en su Decreto 27/ 2015: Atendiendo a sus características, ciertos árboles 
y arboledas singulares incluidos en el Catalogo de árboles y arboledas singulares de Aragón podrán ser declarados Monumento Natural, en cuyo caso, el régimen de protección, será el establecido en la normativa aplicable en materia de monumentos naturales, y en lo que no se oponga a la misma, el previsto en este Decreto. También ayuda plantear, según el caso, otras disposiciones que insten a crear comisiones técnicas y jurídicas interdepartamentales para aquellas situaciones en las que las figuras de protección de una ley recaen sobre bienes o elementos declarados al amparo de otras leyes (como propone la Comunidad de Murcia o la de Valencia, entre otras).

En ocasiones, se logra alcanzar mayor concreción. Aragón es un ejemplo en el que el desarrollo legislativo en cuestión de patrimonio arbóreo ha seguido una evolución más ordenada. Así, lo que comenzó en una "intención" de Catálogo de árboles singulares, es actualmente una realidad que se ha ido conformando sobre el perfeccionamiento en su definición. Sin prescindir de la figura más genérica "monumento natural", que reservaba solo para aquellos ejemplares más sobresalientes, fue ahondando en la categoría de árbol singular (creada en la Ley 8/2004 de 20 de diciembre, de Medidas Urgentes en Materia de Medio Ambiente) hasta su definición en el Decreto 34/2009 donde crea el Catálogo de árboles y arboledas singulares. En este decreto se detallan aspectos (no contemplados en otras definiciones) y se utilizan metodologías como la Norma Granada para la evaluación del arbolado. Llega hasta la ley vigente, Ley 6/2014, donde se afina en la concepción de árbol singular de Aragón. Y se aprueba el Decreto 27/2015, para regular el Catálogo de árboles y arboledas singulares, donde es reconsiderado un nuevo modelo de valoración y donde establece la necesidad de fijar nuevos criterios de valoración de la singularidad de los árboles singulares. A destacar en su régimen jurídico de protección la importancia que otorga al lugar, al entorno del árbol a proteger a través de la delimitación de unos criterios que lo definen. Esto mejora el planteamiento del entorno de protección con respecto al que marcan otros decretos, en base a una superficie fija (por ejemplo, los 3 metros fijos alrededor del área de proyección de la copa como Zona Periférica de Protección que dicta el Decreto 265/1995 de la Comunidad Autónoma del País Vasco).

El País Vasco cuenta con la figura de protección de “Árbol Singular" bajo la que se amparan aquellos árboles "de características extraordinarias que destaquen por razón de su tamaño, edad, historia, belleza o situación” en la Ley 16/ 1994 de Conservación de la Naturaleza del País Vasco que la recoge expresamente. A partir de la misma, se crea el Catálogo de Árboles Singulares de la Comunidad Autónoma del País Vasco por Decreto 265/1995. En apenas dos años, el Catálogo se amplió por Decreto 23/1997 con una segunda declaración de Árboles Singulares, sin embargo, desde entonces no ha vuelto a actualizarse.

Si en Euskadi la figura específica de árbol singular está comprendida en la propia ley, en Cataluña (como en Galicia y Extremadura, entre otras) es necesario que se aprueben los correspondientes decretos que contienen las definiciones concretas para su declaración. Entra, por tanto, en mayor detalle y en una clasificación según características o circunstancias. Así, en este caso, y en base a la Ley 12/1985, 
de Espacios Naturales, la protección de árboles y arboledas monumentales de Cataluña se basa en tres decretos (Decreto 214/1987, 47/1988 y 120/1989) que definen y regulan diferentes tipologías: Árboles monumentales; Árboles de interés comarcal y local; y Arboledas monumentales de interés comarcal y de interés local, respectivamente.

Las figuras expresas de "árbol singular" recogen definiciones muy similares a la que se cita para el País Vasco, incluso procedentes de leyes de conservación de la naturaleza, como se ha visto para otras comunidades autónomas. Ahora bien, en el caso de La Rioja, se puntualiza: vinculación a un momento o paisaje, consideración que podría merecer mayor atención. Se trata de una ley forestal, la Ley 2/1995, de Protección y Desarrollo del Patrimonio Forestal, que alude a la categoría de "árboles singulares" incluyendo a árboles y agrupaciones de árboles en el Inventario de Árboles Singulares de La Rioja. Apoyándose en una Ley de Montes, el desarrollo normativo se aleja, pues, del concepto de "monumento natural" con origen en la Ley de Espacios Naturales. La declaración de árboles singulares y su inclusión en el Inventario llega, tras el Decreto 114/2003 por el que se aprueba el reglamento de desarrollo de la ley, por Orden 3/2006, formalizándose así el Catálogo.

Así pues, muy pocas son las las CC.AA. que poseen una ley específica para la conservación, protección y regulación del patrimonio arbóreo. Esto se da solo en Madrid, la Comunidad Valenciana, Islas Baleares y recientemente, la Región de Murcia. La Comunidad Autónoma de las Islas Baleares es una de las Autonomías pioneras en ello. Con la aprobación de la Ley 6/1991 de Protección de los Árboles Singulares de la Comunidad Autónoma de las Islas Baleares crea el Catálogo de Árboles Singulares en el que se incluyen "todos aquellos árboles de características físicas extraordinarias, interés científico relevante o que sean apoyo de valores culturales señalados". Destaca la Comunidad Autónoma Valenciana con la Ley 4/2006, de Patrimonio Arbóreo Monumental por tratarse de una ley tan detallada. Distingue entre árboles monumentales y árboles singulares a través de un "coeficiente de monumentalidad", definición que debe establecerse mediante decreto que desarrolle esta Ley. En su ordenamiento establece diferentes niveles de protección, según las categorías que alcanzan los ejemplares: expresa por la Generalitat para la declaración de "árboles monumentales" y "árboles singulares"; expresa por los ayuntamientos que declaran "árboles monumentales de interés local"; y la protección genérica (en base a características de perímetro y altura), no formal y sin particularizar. A día de hoy no existe un catálogo formal. Si bien se procedió a la protección directa de numerosos árboles, esto fue por vía de la protección genérica y a través de los ayuntamientos, cuyo catálogo se publicó por Orden 22/2012. Para dotar de protección a otro gran número de árboles que cumplen con los requisitos de monumentalidad, es necesario definir parámetros técnicos y jurídicos más ajustados. Es, por tanto, evidente la urgencia de un reglamento que desarrolle la ley. La Comunidad Autónoma de Murcia se inicia en este proceso, con la Ley 14/2016, de Patrimonio Arbóreo Monumental de la Región de Murcia, de velar por la conservación de sus árboles singulares. Muy similar a la ley de Valencia y en la situación de desarrollar la reglamentación para una protección más efectiva. 
En contraste con varias de las situaciones reflejadas por las comunidades autónomas, el caso de la Comunidad Autónoma de Madrid sirve, quizá, como ejemplo de mayor coherencia. Por un lado, la Ley 2/1991, para la Protección y Regulación de la Fauna y Flora Silvestre, crea el Catálogo Regional de Especies Amenazadas de Fauna y Flora Silvestres. Al amparo de esta ley, se aprueba el Catálogo Regional de Árboles Singulares de la Comunidad de Madrid mediante Decreto 18/1992 (con una actualización por Orden 68/2015), que además crea la categoría de "Árboles Singulares". El decreto recoge menciones específicas y establece la elaboración de "planes de manejo" para aquellos árboles que lo requieran. Por otro lado, la Ley 8/2005, de Protección y Fomento del Arbolado Urbano ha sido pionera en incorporar un programa para la protección especial de algunos ejemplares y la conservación del arbolado urbano en general. En su consideración del árbol como "parte del patrimonio histórico-artístico de la ciudad" lo dota de una protección, al margen de la que existe por normativa de los distintos municipios de la Comunidad, para asegurar "un tratamiento uniforme a toda su variedad tipológica". En sus Disposiciones Adicionales, contempla un Régimen de los Árboles Singulares, referido a los árboles urbanos incluidos en el Catálogo Regional de Especies Amenazadas de Flora y Fauna Silvestres, dentro de la categoría de Árboles Singulares. Y además un Régimen de los árboles incluidos en los bienes que integran el Patrimonio Histórico de la Comunidad de Madrid. Este consenso entre lo que dictan ambas leyes correspondientes a la Comunidad de Madrid muestra cierta lógica en la práctica, al margen de otras dificultades, hacia la que debería tender la gestión del patrimonio arbóreo.

\section{Conclusiones}

El marco jurídico analizado muestra que la normativa autonómica no alcanza un consenso en cuanto al tratamiento de la figura de "árbol singular" y su definición. La ambigüedad del término con el que se nombra a los árboles singulares da lugar a confusiones que dificultan los procesos de evaluación y de catalogación. La norma disminuye su efectividad, lo que repercute en la garantía de una protección final. No se trata de formular una definición universal pues la realidad territorial difiere de una comunidad autónoma a otra. Por otro lado, cada árbol se caracteriza por unas condiciones y particularidades que le son propias y que es preciso conocer.

En la revisión de las diversas definiciones aportadas por la legislación autonómica referida a la protección del arbolado, se constata que, en la mayor parte de las autonomías, si el cuerpo legal cuenta con una figura específica para la consideración del árbol singular, la categoría se ve duplicada por la existencia de otras figuras de protección referidas a la conservación de la naturaleza, lo que induce a diferentes interpretaciones y confusión por multiplicidad de voces.

Se hace evidente que, en general, la definición que contiene la figura expresa de árbol singular carece de precisión. El concepto de árbol singular se formula en 
un enunciado que suele contener aspectos, necesarios para el reconocimiento del valor del árbol, en los que no se profundiza. Por otro lado, se observa que las definiciones jurídicas, en general, destacan los aspectos más descriptivos y funcionales del árbol. La dimensión, la edad y la rareza del árbol se presentan como primeros atributos por los que se reconoce la singularidad del ejemplar, obviando, en ocasiones, otro tipo de características referidas al significado cultural, especialmente en lo que se refiere al entorno o al paisaje.

Por todo lo dicho, se requiere abordar la revisión y actualización del marco legislativo a nivel autonómico. Es necesario ahondar en esta cuestión, averiguar en qué criterios se basa cada Comunidad Autónoma o región para definir a sus árboles singulares y de interés. Esto ayudaría a localizar vacíos en la nomenclatura y en el contenido de la legislación. Además, convendría revisar los parámetros de caracterización del arbolado para un mayor conocimiento en detalle y, por tanto, para una idea más aproximada de su verdadera importancia, de su evaluación.

\section{Bibliografía}

Caro Baroja, J., 1946. Olentzaro. La fiesta del solsticio de invierno en Guipúzcoa oriental y algunas localidades de la Montaña navarra, Revista de dialectología y tradiciones populares, 1946, II. Madrid: 1946; pp. 42-68.

Ceballos, L. \& Ruiz de La Torre, J., 1979. Árboles y arbustos de la España peninsular. Fundación Conde del Valle de Salazar, Instituto Forestal de Investigaciones y Experiencias y Escuela Técnica Superior de Ingenieros de Montes. Madrid.

Cuenca, C., Melero, M., Cortina, J., 2016. Análisis de las políticas de restauración forestal en España (1983-2013), Cuadernos Sociedad Española Ciencias Forectales 42 (2016) 61-74.

Domínguez, S. (coor.) et al.2009. Gigantes y ancianos de los bosques españoles: una búsqueda de los tesoros vivientes de nuestro territorio. Ed. Bosques Sin Fronteras, Madrid.

Espluga, A., Vigil, D., Marín, J., 2001. Estudio comparativo de la legislación medioambiental de Espacios Naturales, Observatorio Medioambiental, Norteamérica, n. ${ }^{\circ}$ 4, 2001. Págs. 151-167.

Florido, G., Lozano, P.J., 2005. Las figuras de protección de los espacios naturales en las comunidades autónomas españolas: una puesta al día. Boletín de la A.G.E. n. ${ }^{\circ} 40$ 2005 , págs. $57-81$

Hallé, F., 2014. Plaidoyer pour l'arbre. Actes Sud, Arles [1re édition, 2005]

Hallé, F., 2011. La vie des arbres. Collection Les petites conférences. Bayard Éditions.

Kleinn, C., 2000. Inventario y evaluación de árboles fuera del bosque en grandes espacios. Unasylva, n. ${ }^{\circ} 200$, Los árboles fuera del bosque. Revista internacional de silvicultura $\mathrm{e}$ industrias forestales, Vol. 51- 2000/1. FAO.

Lafuente, M., 2007. Concepto y protección del patrimonio arbóreo monumental. Revista de Administración Pública, 172, 403-437.

Pérez-Soba, I. y Picos, J., 2000, Antecedentes de la protección legal de los árboles singulares en España, Montes: Revista de Ámbito Forestal, n. ${ }^{\circ}$ 60, pp. 72-80. 
Ramos, A., (coord.), 1987. Diccionario de la naturaleza. Hombre, ecología y paisaje, Espasa-Calpe S.A., Madrid

Villota, M., 2015. Los árboles singulares en el paisaje. Propuesta de un modelo para su evaluación: el caso del territorio histórico de Álava. Tesis Doctoral, Universidad Politécnica de Madrid.

Villota, M., Escribano, R., 2015. Metodología para una valoración del arbolado singular del territorio histórico de Álava. En: De la Riva, J., Ibarra, P., Montorio, R., Rodrigues, M. (Eds.), Análisis espacial y representación geográfica: innovación y aplicación. 12711280. Universidad de Zaragoza-AGE. 
